\title{
Effects of 20-Week Intermittent Cold-Water-Immersion on Phenotype and Myonuclei in Single Fibers of Rat Hindlimb Muscles
}

\author{
J.H. LEE*, E.Y. HAN*, M.S. KANG*, F. KAWANO ${ }^{\dagger \ddagger}$, H.J. KIM*, Y. OHIRA ${ }^{\dagger \ddagger}$, and C.K. KIM* \\ *Human Physiology, Korea National Sport University, Seoul, Korea; and ${ }^{\dagger}$ School of Health and Sport Sciences and \\ ${ }^{\ddagger}$ Graduate School of Medicine, Osaka University, Toyonaka, Osaka, 560-0043 Japan
}

\begin{abstract}
The effects of 20 weeks of intermittent cold-water-immersion on myosin heavy chain (MHC) expression, cross-sectional area (CSA), myonuclear number, and myonuclear domain size in isolated single fiber of soleus and extensor digitorum longus (EDL) muscles were studied in male Wistar rats. Cold exposure was accomplished by submerging the rats in shoulder-deep water, maintained at $\sim 18^{\circ} \mathrm{C}$, for 1 hour/day, 5 days/week and for 20 weeks. Cold exposure resulted in a significant inhibition of body and soleus muscle weight gain. The percent type Ila MHC fibers of EDL muscle was increased, whereas that of type Ila $+\mathrm{b} \mathrm{MHC}$
\end{abstract}

fibers was less in cold-exposed group than controls $(p<0.05)$. The mean CSA and myonuclear number in type I MHC fibers of soleus muscle in cold-exposed group were significantly less than controls. Myonuclear domain in type Ila fibers of EDL in the cold-exposed group was greater than controls $(p<0.05)$. It is suggested that prolonged cold exposure causes the fibertype-specific adaptation in rat hindlimb muscles. It is further indicated that cold-exposure-related modulation of myonuclear number was closely related to reduction of fiber CSA, not the shift of fiber phenotype. [The Japanese Journal of Physiology 54: 331-337, 2004]

Key words: cold exposure, cross-sectional area, myosin heavy chain expression, myonuclear number and domain, rat skeletal muscle fibers.

It has been reported that an adaptation of the metabolic and contractile properties of skeletal muscles is induced in response to cold exposure [1-3]. An improved fatigue resistance of fast-twitch extensor digitorum longus (EDL) muscle, but not of slowtwitch soleus muscle, in response to train stimulation at $10 \mathrm{~Hz}(100 \mathrm{~ms}$ train duration, 60 trains/min) was induced following 20 -week intermittent cold-water immersion in rats [1]. It is also reported that mitochondrial enzyme activities, which influence the fatigue resistance, in fast-twitch gastrocnemius muscle of frog were increased in response to chronic cold exposure at $4^{\circ} \mathrm{C}$ [2]. Furthermore, our recent study demonstrated that chronic intermittent cold-water immersion caused a reduction of fiber size in human skeletal muscle [4].
The physical locomotion using hindlimb muscles, which could be one of the causes for the improvement of fatigue resistance in skeletal muscle, was even inhibited during cold exposure. But the high-energy phosphate contents in the muscles were lowered. A similar reduction of high-energy phosphate contents in skeletal muscles was induced by feeding creatine analogue $\beta$-guanidinopropionic acid ( $\beta$-GPA) to rats, and the fatigue resistance during swimming [5] or in response to the in situ electrical stimulation of skeletal muscle [6] was improved. These results suggest that the cold-exposure-associated improvement of fatigue resistance of skeletal muscles may be related to the metabolic stimulation caused by lowered high-energy phosphate levels.

It is well known that the contractile properties of

Received on March 19, 2004; accepted on June 21, 2004

Correspondence should be addressed to: Chang K. Kim, Ph.D., Human Physiology, Korea National Sport University, Olympic Park, 88-15 Oryun-dong, Songpa-gu, Seoul, Korea. Fax: + 82-2-418-1877, E-mail: ckkim@knsu.ac.kr 
skeletal muscles are closely related to the phenotypes of fibers that compose the muscles. The fiber type composition of rat EDL muscle with improved fatigue resistance following $\beta$-GPA feeding was shifted toward a slow-twitch type relative to the controls $[5,6]$. Therefore, it is speculated that cold exposure, which causes the decrease of high-energy phosphate contents, may also result in the shift of fiber characteristics toward a slow-twitch type. However, Walters and Constable [3] demonstrated that chronic cold $\left(\sim 20^{\circ} \mathrm{C}\right)$ water immersion induced a fiber type transformation from type I to II in soleus, but had no influence in EDL. Thus, it is still unclear how the fiber phenotypes in rat skeletal muscles are influenced by cold exposure.

Skeletal muscle fibers are multinucleated cells containing thousands of myonuclei. It is reported that the number of myonuclei or myonuclear domain (cytoplasmic volume per myonucleus) is influenced by the activity level of muscle. For example, the myonuclear number in hypertrophying muscle fibers was increased [7-9] and that in fibers associated with atrophy was decreased in response to the exposure to microgravity environment [10]. The unloadingrelated atrophy is generally associated with a shift of fiber phenotype toward the fast-twitch type [11]. However, it is not known whether metabolic stimuli in response to cold exposure influence the myonuclear number.

Therefore, the current study was performed to investigate the responses of the cross-sectional area (CSA), phenotype, myonuclear number, and myonuclear domain in fibers of both fast- and slow-twitch muscles of rats to chronic intermittent cold exposure.

\section{MATERIALS AND METHODS}

\section{Animal care and experimental procedures.}

All experimental procedures were conducted in accordance with the Japanese and Korean Physiological Society Guide for the Care and Use of Laboratory Animals. The study was also approved by the Committee on Animal Care and Use at the university. Ten male Wistar rats (Kyudo, Kumamoto, Japan) with mean $( \pm$ SEM) initial body weight of $91.3 \pm 4.6$ $\mathrm{g}$ were used. All rats were housed individually in stainless steel cages $(29 \times 40 \times 30 \mathrm{~cm})$ and were pair-fed a powdered diet (CE-2, Nihon CLEA, Tokyo). The daily food supply was gradually increased following growth, and each rat was supplied $20 \mathrm{~g}$ diet daily from week 4 to the end of the experiment. Water was given ad libitum. The temperature and humidity of the animal room with 12:12-hour light (6 a.m.-6 p.m.): dark
(6 p.m.-6 a.m.) cycle were maintained at $\sim 23^{\circ} \mathrm{C}$ and $\sim 50 \%$, respectively.

Rats were randomly assigned to control or a coldexposed group ( $n=5$ each). Cold exposure was accomplished by submerging the rats in shoulder-deep water during the light period, using a plastic cylinder with $50 \mathrm{~cm}$ diameter and $60 \mathrm{~cm}$ height. The water temperature was maintained at $\sim 18^{\circ} \mathrm{C}$. Initially, the rats were exposed to cold water for $5 \mathrm{~min} /$ day. The exposure time was increased 5 min each day until the final period of $60 \mathrm{~min} /$ day was reached. At the end of exposure, the water was wiped off immediately. The cold-exposed rats received this treatment 5 days/week for 20 weeks.

After 20 weeks of cold-water immersion, the animals were sacrificed with an over-dose i.p. injection of sodium pentobarbital following an overnight recovery from the previous immersion. The soleus and EDL muscles were excised from the left limb and weighed. The muscles were frozen in liquid nitrogen and stored at $-80^{\circ} \mathrm{C}$ until analyzed. The muscles from the right limb were used for analyses of contractile properties [1].

Single fiber isolation. Frozen muscles and vials with 50\% glycerol and 50\% low-calcium relaxing solution [12] were placed in a $-20^{\circ} \mathrm{C}$ Cryostat for several hours to equilibrate to this temperature. The muscle samples were placed in the vials of relaxing solution and stored overnight in a $-5^{\circ} \mathrm{C}$ freezer. In the following morning, the vials were transferred to a refrigerator for 2 hours, and the muscle samples were pinned to a Sylgard-coated culture dish in chilled $100 \%$ relaxing solution for $20-30 \mathrm{~min}$ at room temperature. Single muscle fiber segments ( 50/muscle) were mechanically isolated from muscle sample by the use of a micro-dissection instrument under a microscope [10]. The samples were placed on gelatincoated slide glasses and stored at $-20^{\circ} \mathrm{C}$ until use.

Analysis of myonuclear number. Single-fiber segments to be analyzed were removed from the freezer, thawed, and air-dried for $5 \mathrm{~min}$. Each fiber was circled with a hydrophobic pen barrier (Kiyota International, Elk Grove Village, IL, U.S.A.) and rinsed briefly in phosphate buffered saline (PBS) for $5 \mathrm{~min}$. The Fibers were stained for $5 \mathrm{~min}$ with $54 \mu \mathrm{M}$ acridine orange in PBS and then for 5 min with 1.5 $\times 10^{-7} \mathrm{M}$ propidium iodide in PBS. This combination was found to produce the best staining of, and contrast between, the cytoplasm and the myonuclei. After the fibers were stained, they were rinsed with PBS and mounted in $100 \%$ glycerol with cover slips with "struts" of hardened nail polish on the corners to minimize fiber compression. A Fluoview Confocal 
Microscopy (Olympus, Tokyo, Japan) was used to analyze fiber CSA, myonuclear number, and cytoplasmic volume per myonucleus.

First, a series of scans was taken through the entire $\mathrm{Z}$ thickness of a fiber region with the proper filter sets for acridine orange fluorescence. The myonuclear number was determined by counting all the myonuclei in the stack for this region and converted into myonuclei per millimeter by dividing by the length of the field $(128 \mu \mathrm{m})$ and multiplying by 1,000 .

Next, maximum-intensity projection rotated orthogonally to the long axis of the fiber was produced from the stack, and fiber CSA was measured with calibrated measurement software (Olympus, Tokyo, Japan). To correct for possible effects of the different states of fiber stretch, 10 consecutive sarcomere lengths of the fiber were measured. This was done in three different regions, and the results were averaged. Both myonuclei per millimeter and CSA were corrected for differences in sarcomere length by multiplying by the observed sarcomere length and dividing by 2.5 to normalize to a $2.5 \mu \mathrm{m}$ sarcomere length.

The average cytoplasmic volume per myonucleus was then calculated by multiplying fiber CSA by the length of the fiber region $(128 \mu \mathrm{m})$ and dividing by the number of myonuclei counted in that region. For each fiber, three non overlapping regions were randomly chosen along the fiber length and analyzed. Data from all three regions were pooled and averaged to obtain a single value for the fiber. Fiber ends, damaged regions, and excessively stretched (sarcomere length $>3.5 \mu \mathrm{m}$ ) regions were omitted from the analyses.

Single-fiber gel electrophoresis. After confocal analysis, gel electrophoresis was carried out in the same single fiber segments. Briefly, single fibers were unmounted, rinsed free of glycerol with PBS, dehydrated, and destained in 50\% ethanol for $5 \mathrm{~min}$. Fibers were air-dried for $5 \mathrm{~min}$, scraped off the slide with a clean razor blade, and placed in a microcentrifuge tube containing $10 \mu \mathrm{l}$ of $1 \mathrm{x}$ electrophoresis sample buffer [13]. Fibers in sample buffer were stored at $-5^{\circ} \mathrm{C}$ until electrophoresis was performed.

Electrophoresis was carried out with a Bio-Rad Mini-Protean II dual-slab electrophoresis unit (Richmond, CA, U.S.A.) as described by Talmadge and Roy [14]. Separating gels contained 8\% acrylamide and $30 \%$ glycerol to enhance the migration and separation of myosin heavy chain (MHC) isoform. Seven microliters of each sample was loaded in a single gel lane. To facilitate an accurate determination of MHC isoform expression, two "stan- dard" lanes were loaded with $10 \mu \mathrm{l}$ of a mixture of homogenate of rat medial gastrocnemius and soleus muscles. The gels were run overnight at $80 \mathrm{~V}$ in an ice-packed cooler. They were stained with rapid Coomassie (Diversified Biotech, Boston, MA, U.S.A.). The fiber phenotypes were classified into slow-twitch pure type I MHC and fast-twitch pure type IIa and hybrid type I + IIa MHC in soleus fibers. Those in EDL were classified into pure type I, IIa, and IIb MHC and hybrid type I + IIa and IIa + b MHC.

Statistical analyses. All values were expressed as mean \pm SEM. Significant differences between each group were determined by an analysis of variance followed by Scheffe's post hoc test. Statistical significance was accepted at $p<0.05$.

\section{RESULTS}

Body and muscle weights. Body weight in both the control and cold-exposed groups gradually increased during the experimental period (Fig. 1). But the mean levels of the cold-exposed group were significantly less than those of control during weeks 8 and 20, even though the amount of daily food intake was identical. The absolute soleus weight at the end of 20 weeks of cold exposure was significantly less than that of the control group (12\%, Fig. 2). The same trend was observed in EDL muscle $(p>0.05)$. The weight relative to body weight, however, tended to be increased following cold exposure. The weight in EDL of the cold-exposed group was significantly greater than of the control group.

Fiber phenotype and cross-sectional area. The percent of fiber phenotypes in soleus was not influenced significantly by cold exposure, although the fibers expressing pure type IIa MHC, which was seen in the control muscle, were not observed in the cold-exposed group (Fig. 3). In EDL muscle, the percent of pure type IIa MHC fiber was increased by

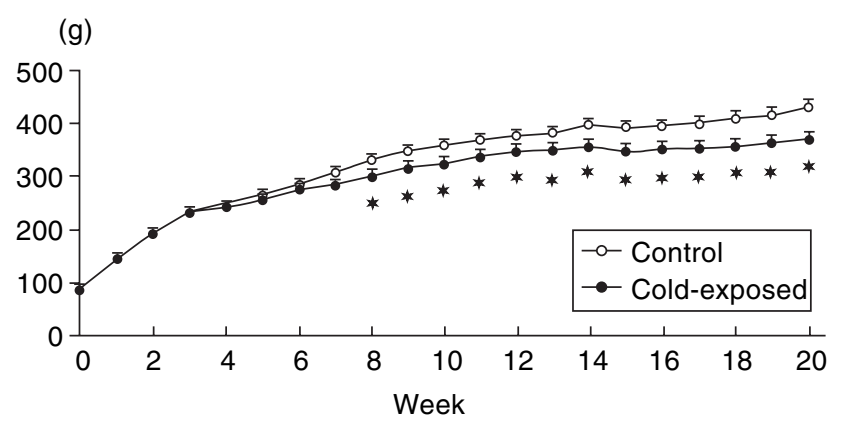

Fig. 1. Changes in body weight during a 20-week experimental period. $n=5$ in each group. Mean \pm SEM. *: $p<0.05$ vs. control. 

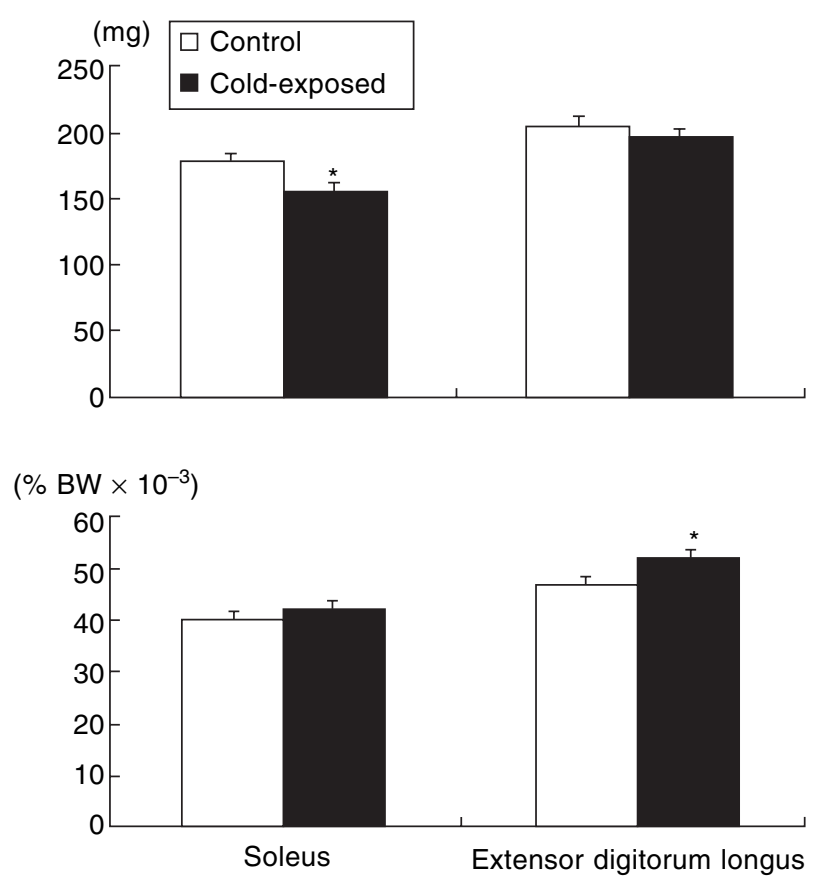

Fig. 2. Absolute ( $\mathrm{mg}$ ) and relative (\% BW) wet weight of soleus and extensor digitorum longus. $n=5$ in each group. Mean \pm SEM. ${ }^{*}: p<0.05$ vs. control. BW: body weight.
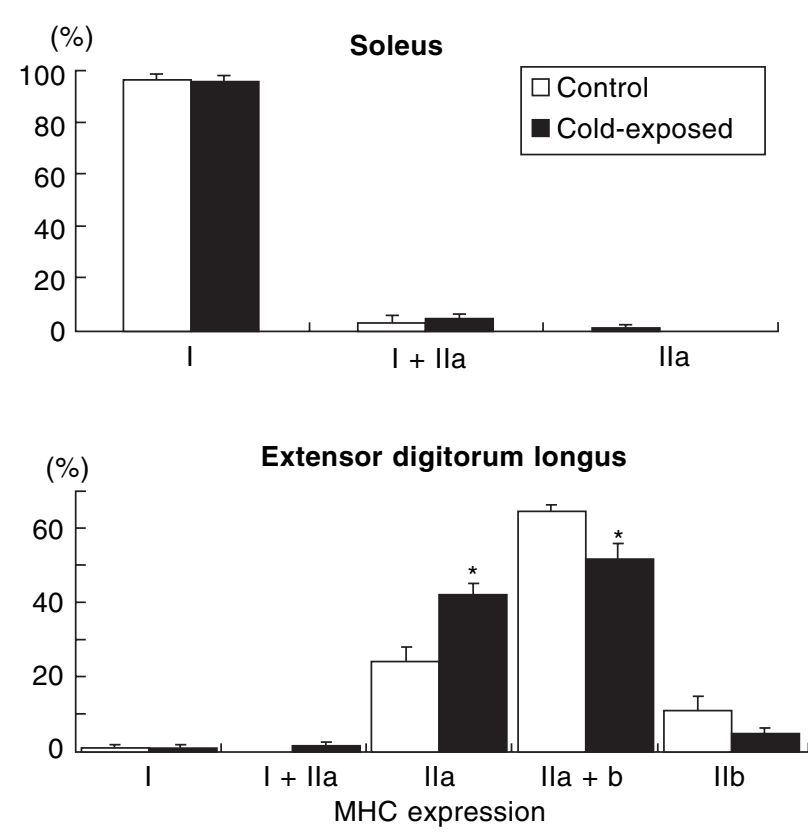

Fig. 3. Percent distribution of fiber phenotype in soleus and extensor digitorum longus. $n=5$ in each group. The mean of $\sim 50$ fibers were used for each muscle. Mean \pm SEM. * $: p<0.05$ vs. control. MHC: myosin heavy chain.

cold exposure $(p<0.05)$. And the percent of type IIa $+\mathrm{b}$ MHC $(p<0.05)$ and type IIb MHC fibers $(p>$ 0.05 ) in the cold-exposed group were less than controls. De novo appearance of fibers expressing type I + IIa MHC was noted in response to cold exposure.

The responses of muscle fiber size to cold expo- sure are shown in Fig. 4. The mean CSA in the soleus fibers expressing pure type I MHC of the coldexposed group was significantly less than controls. Although there was also the same trend in fibers expressing type I + IIa MHC, the difference between the two groups was not statistically significant. Fiber CSA in EDL was not significantly affected by cold exposure.
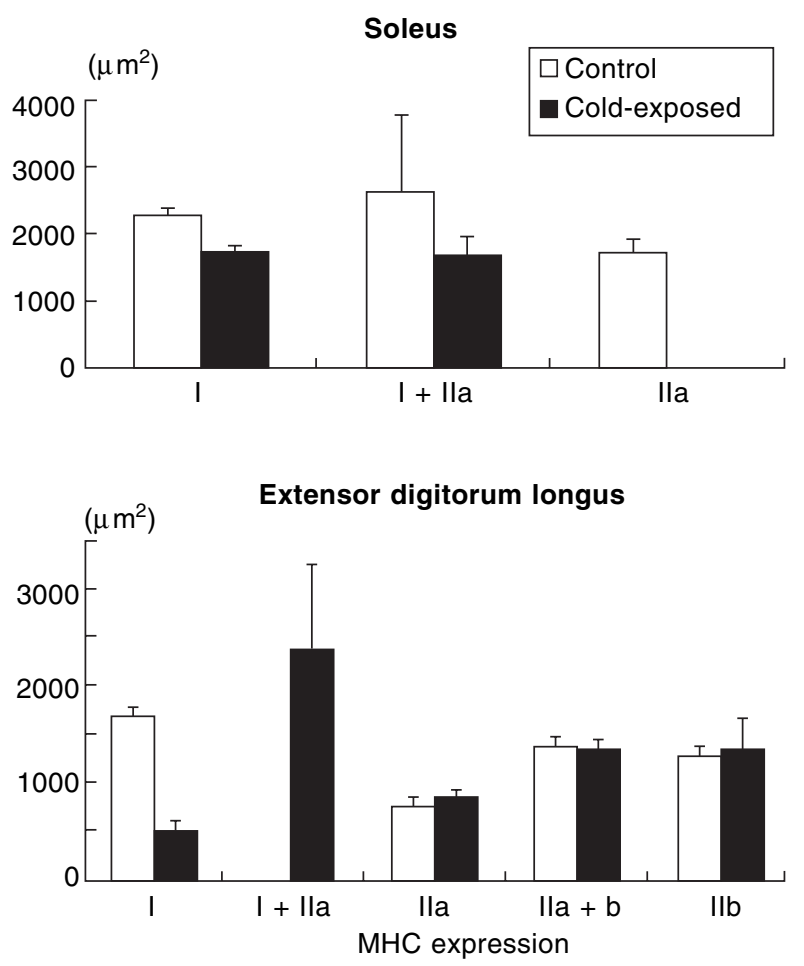

Fig. 4. Cross-sectional area in each type of fibers of soleus and extensor digitorum longus. $n=5$ in each group. The mean of $\sim 50$ fibers were used for each muscle. Mean \pm SEM. ${ }^{*}: p<0.05$ vs. control. MHC: myosin heavy chain.

Myonuclei. The mean myonuclear number $/ \mathrm{mm}$ of fiber length in pure type I MHC fibers of soleus was significantly less in the cold-exposed group than in the control group (Fig. 5). The number in type I+ IIa MHC fibers also tended to decrease in response to cold exposure $(p>0.05)$. The myonuclear number in EDL fibers was not affected by cold exposure. The size of myonuclear domain in EDL fibers expressing pure type IIa MHC in the cold-exposed group was also significantly greater than in controls (Fig. 6). However, the levels in other types of fibers were not significantly different between the two groups.

\section{DISCUSSION}

Body weight and muscle mass. The growthrelated gain of body and skeletal muscle weight was inhibited by 20 weeks of intermittent cold exposure. 

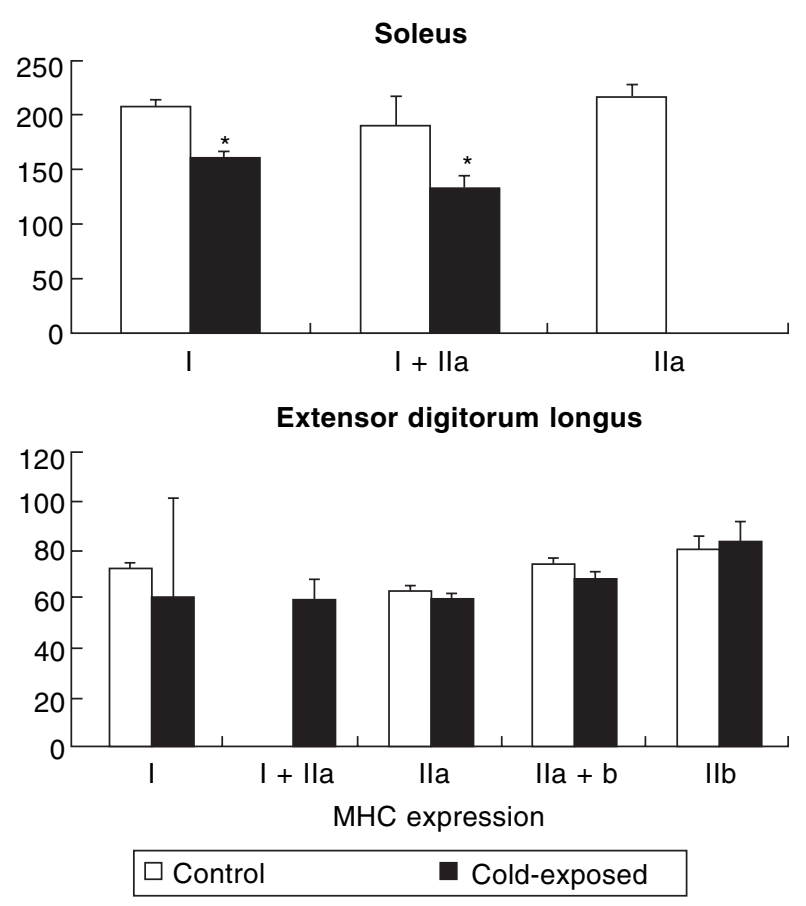

Fig. 5. Myonuclear number per millimeter of length in each type of fibers of soleus and extensor digitorum longus. $n=5$ in each group. The mean of $\sim 50$ fibers were used for each muscle. Mean \pm SEM. MHC: myosin heavy chain.
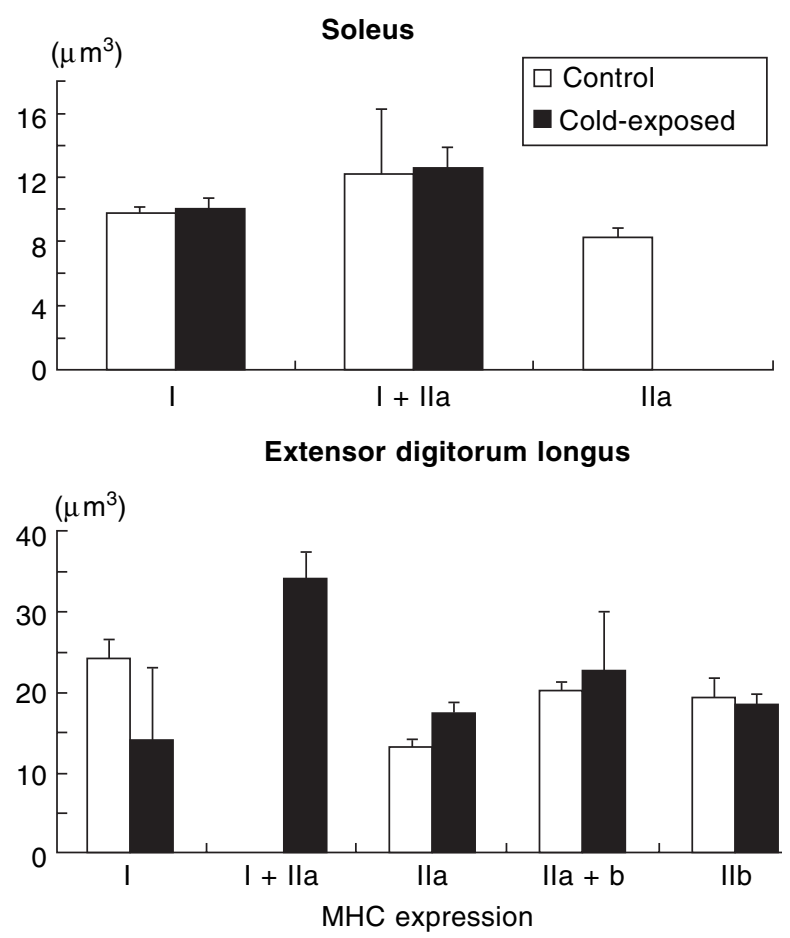

Fig. 6. Myonuclear domain (cytoplasmic volume per myonucleus) in each type of fibers of soleus and extensor digitorum longus. $n=5$ in each group. The mean of $\sim 50$ fibers was used for each muscle. Mean \pm SEM. *: $p<0.05$ vs. control. MHC: myosin heavy chain.
However, the muscle weight normalized by body weight was even increased generally following cold exposure, suggesting that the cold-exposure-related inhibition of the gain was greater in whole body weight than that in each skeletal muscle. The responses of body and muscle weights to cold exposure are comparable to those reported elsewhere $[3,15,16]$.

Chronic cold exposure causes a drop of body temperature $[3,15,17]$. To raise or maintain body temperature, thermogenesis is stimulated. Askew [17] reported that body weight loss was common during chronic exposure to cold environment, because cold exposure elevated energy expenditure almost 2.5 times over the subjects in the warm environment. Such phenomena were also supported by the findings that cold exposure resulted in an increased interscapular brown adipose tissue mass and plasma catecholamine levels in rats [1].

Mount [18] reported that the main source of thermogenesis was from food intake, and rats exposed to cold environment generally increase the volume of food intake $[3,15,17]$. Therefore, the rats were pairfed to avoid the effects of different amounts of food intake on the responses of skeletal muscles in the current study. The effects of cold exposure on body and muscle weights were similar to those of the studies, in which the rats were not pair-fed [3]. It is suggested that the cold-exposure-associated inhibition of body and muscle weight gain is not directly related to the amount of food intake.

The inhibition of muscle growth was more prominent in slow-twitch soleus than in fast-twitch EDL. Similar results were also reported elsewhere [1]. The mean CSA of single muscle fibers expressing pure type I MHC in the soleus of the cold-exposed group was also significantly less than in controls. A similar phenomenon was noted in type I MHC fibers of EDL, even though statistical significance was not observed because the distribution of this type of fibers was very low. The responses of fiber size observed in the present study are comparable with those reported by others [4, 19]. Samuels et al. [16] found that 10 days of cold exposure decreased the growth rate because of a lower rate of protein synthesis, instead of an increased rate of protein degradation. However, the mean sizes of all muscle fibers with different phenotypes in sea divers who have been diving $4-5 \mathrm{~h}$ daily during their life in water with temperatures ranging from $10-12^{\circ} \mathrm{C}$ to $25-27^{\circ} \mathrm{C}$ during the winter and summer, respectively, were smaller than those in non-divers [4]. Especially, the size of fast-twitch fibers co-expressing types IIa and IIx MHC was markedly smaller than type I MHC fiber in these 
divers. Nevertheless, it is still unclear why the slowtwitch muscle fibers are more susceptible to cold exposure.

Fiber phenotype. Previous studies have demonstrated that the shift of skeletal muscle fiber type could occur following cold acclimation in fishes [20,21] and mammals [3]. Walters and Constable [3] reported that the submerging of rats in shoulder-deep water maintained at $20^{\circ} \mathrm{C}$, for $1 \mathrm{hr} /$ day, 5 days/week for 17-19 weeks caused a type I to type II fiber transformation in soleus, while having no influence on EDL. However, a cold-exposure-related shift of fiber type from IIa $+b$ MHC to IIa MHC was induced in EDL, not in soleus, in the present study. Even though it is unclear why such a discrepancy exists, differences in the species and age may influence the response to cold exposure. Walter and Constable [3] used Sprague Dawley rats with an_initial body weight of $\sim 353 \mathrm{~g}$, and we used Wistar rats with a body weight of $\sim 91 \mathrm{~g}$. Further, one of the other factors may be the difference in water temperatures. The temperature they used was $\sim 20^{\circ} \mathrm{C}$, but that in the current study was $\sim 18^{\circ} \mathrm{C}$.

Pette and Staron [22] indicated that the expression of myosin genes in skeletal muscle could be regulated by several factors, including neural innervation, hormonal action, electrical stimulation, and mechanical activity. For instance, an electrical stimulation at a low frequency [22] and endurance training [23-25] could increase the distribution of type I MHC fibers in rat skeletal muscle. Pette and Staron [22] also described that MHC transitions appear to reflect an obligatory pathway of MHC gene expression to the following order of MHC I MHC IIa MHC IIx MHC IIb. A decreased distribution of type IIa $+b$ MHC fibers and increased type IIa MHC fibers of EDL seen in the current study may be influenced by such transition of MHC expression. A significant improvement of fatigue resistance in response to in vitro train stimulation at $10 \mathrm{~Hz}(100 \mathrm{~ms}$ train duration, and 60 trains/min) was observed in EDL sampled from the contralateral right limb of the same rat as in the present study [1]. Therefore, the results obtained in the current study and in the measurement of contractile properties agree and suggest that a shift of fiber phenotype from type II $a+b$ MHC to IIa MHC and improved fatigue resistance were caused by cold exposure.

Myonuclei. There are more myonuclei in slow fibers than in fast fibers in control rats [7, 26, 27]. A significant decrease in the myonuclear number in response to cold exposure was noted in type I MHC fibers of soleus, in which the percent distribution of fiber type was stable. However, the myonuclear number of EDL muscle fibers, in which the percent distribution of fiber phenotype was changed, was not influenced by cold exposure. We also found that the number of myonuclei in the muscle fibers of $\beta$-GPAfed rats was unchanged, even though the fiber phenotype was significantly shifted toward the slowtwitch type (unpublished observation). Therefore, these data suggest that the reduction of myonuclear numbers in response to cold exposure was not directly related to the shift of fiber phenotype.

It is also reported that a decreased myonuclear numbers was observed in atrophied muscle fibers following spaceflight [10], hindlimb suspension [28], or reduction in neuromuscular activity due to spinal isolation [7]. The mean CSA of the fibers with a decreased myonuclear number in cold-exposed soleus was significantly less than that of controls. Further, the responses of CSA (Fig. 4) and myonuclear number (Fig. 5) were generally similar in most fibers expressing various types of MHC in both soleus and EDL. These results suggest that the cold-exposurerelated decrease in the distribution of myonuclei is closely associated with the reduction of fiber size.

There is no published evidence indicating how the myonuclear number is modulated in response to cold exposure. It is suggested that protein synthesis, especially in type I MHC fibers of soleus, may be inhibited following cold exposure, since fiber CSA was significantly less than controls in the present study. However, it is reported that cold exposure stimulates the synthesis of mitochondrial proteins, on the contrary [2]. The specific activities of mitochondrial enzymes, such as cytochrome oxidase, succinate dehydrogenase, and citrate synthase, in frog gastrocnemius muscle were stimulated following chronic cold exposure. Allen et al. [7] reported that the reduction of the myonuclear number may be related to apoptosis, which is induced after gravitational unloading. However, it is still unclear how the cold-exposurerelated decrease in the myonuclear number is modulated.

Conclusion. The effects of 20 weeks of intermittent cold exposure on MHC expression, CSA, the number of myonuclei, and myonuclear domain size in soleus and EDL muscle fibers were studied in male Wistar rats. Cold exposure resulted in a significant inhibition of a growth-related gain of muscle weight and an increase of CSA in pure type I MHC fibers of soleus, not in EDL. The number of myonuclei in these fibers of soleus was also less than in controls. A significant shift of fiber phenotype was induced only in EDL. The percent distribution of fibers 
expressing type IIa $+b$ MHC decreased and that of type IIa MHC was increased following cold exposure. However, the myonuclear number and domain in these fibers were stable. It is suggested that the cold-exposure-related modulation of myonuclear number was closely related to the reduction of fiber CSA, not to the shift of fiber phenotype.

This study was, in part, supported by a Grant-in-Aid for Scientific Research (A, 15200049, Y.O.) from the Japan Society for the Promotion of Science and Grant-in-Aid for Young Scientists (B, 15700417, F.K.) from the Ministry of Education, Culture, Sports, Science and Technology.

\section{REFERENCES}

1. Nomura T, Kawano F, Kang MS, Lee JH, Han EY, Kim CK, Sato Y, and Ohira Y: Effects of long-term cold exposure on contractile muscles of rats. Jpn J Physiol 52: 85-93, 2002

2. Ohira M, and Ohira Y: Effects of exposure to cold on metabolic characteristics in gastrocnemius muscle of frog (Rana pipiens). J Physiol (Lond) 395: 589-595, 1988

3. Walters TJ, and Constable SH: Intermittent cold exposure causes a muscle-specific shift in the fiber type composition in rats. J Appl Physiol 75: 264-267, 1993

4. Bae KA, Ahn NY, Kwon YW, Kim C, Yoon JS, Park SC, and Kim CK: Muscle fiber size and capillarity in Korean diving women. Acta Physiol Scand 179: 167-172, 2003

5. Ohira Y, Kawano F, Roy RR, and Edgerton VR: Metabolic modulation of muscle fiber properties unrelated to mechanical stimuli. Jpn J Physiol 53: 389-400, 2003

6. Wakatsuki T, Ohira Y, Nakamura K, Asakura T, Ohno $\mathrm{H}$, and Yamamoto $\mathrm{M}$ : Changes of contractile properties of extensor digitorum longus in response to creatine-analogue administration and/or hindlimb suspension in rats. Jpn J Physiol 45: 979-989, 1995

7. Allen DL, Monke SR, Talmadge RJ, Roy RR, and Edgerton VR: Plasticity of myonuclear number in hypertrophied and atrophied mammalian skeletal muscle fibers. J Appl Physiol 78: 1969-1976, 1995

8. Cabric M, and James NT: Morphometric analyses on the muscles of exercise trained and untrained dogs. Am J Anat 166: 359-368, 1986

9. Winchester PK, and Gonyea WJ: A quantitative study of satellite cells and myonuclei in stretched avian slow tonic muscle. Anat Rec 232: 369-377, 1992

10. Allen DL, Yasui W, Tanaka T, Ohira Y, Nagaoka S, Sekiguchi C, Hinds WE, Roy RR, and Edgerton VR: Myonuclear number and myosin heavy chain expression in rat soleus muscle fibers after spaceflight. J Appl Physiol 81: 145-151, 1996

11. Ohira $Y$, Jiang $B$, Roy RR, Oganov V, Ilyina-Kakueva E, Marini JF, and Edgerton VR: Rat soleus muscle fibers responses to 14 days of spaceflight and hindlimb suspension. J Appl Physiol 73 (Suppl): 51S-57S, 1992

12. Donaldson SKB: Calcium-activated force-generating properties of mammalian skeletal muscle fibers: his- tochemically identified single peeled rabbit fibers. J Muscle Res Cell Motil 5: 593-612, 1984

13. Laemmili UK: Cleavage of structural proteins during the assembly of the head of bacteriophage T4. Nature 227: 680-685, 1970

14. Talmadge RJ, and Roy RR: Electrophoretic separation of rat skeletal muscle myosin heavy chain isoforms. $J$ Appl Physiol 75: 2337-2340, 1993

15. Owen TL, Spencer RL, and Duckles SP. Effect of age on cold acclimation in rats: metabolic and behavioral responses. Am J Physiol 260 (Regulatory Integrative Comp Physiol 29): R284-R289, 1991

16. Samuels SE, Thompson JR, and Christopherson RJ: Skeletal and cardiac muscle protein turnover during short-term cold exposure and rewarming in young rats. Am Journal Physiol 270 (Regulatory Integrative Comp Physiol 39): R1231-R1239, 1996

17. Askew EW: Nutrition and performance at environmental extremes. In Wolinsky I and Hickson JF (Eds), Nutrition in Exercise and Sport (2nd ed), CRC press, Boca Raton, Florida: 455-474, 1994

18. Mount LE: Effect of climate on the heat exchanges of men and farm animals. In Food, Nutrition and Climate. London: Applied Science: 215-224, 1981

19. Johnston IA, Fleming JD, and Crockford T: Thermal acclimation and muscle contractile properties in cyprinid fish. Am J Physiol 259 (Regulatory Integrative Comp Physiol 28): R231-R236, 1990

20. Gerlach G.-F, Turay L, Malik KTA, Linda J, Scutt A, and Goldspink G: Mechanisms of temperature acclimation in the caup: a molecular biology approach. Am J Physiol 259 (Regulatory Integrative Comp Physiol 28): R237-R244, 1990

21. Vornanen M: Seasonal and temperature-induced changes in myosin heavy chain composition of crucian carp hearts. Am J Physiol 267 (Regulatory Integrative Comp Physiol 36): R1567-R1573, 1994

22. Pette D, and Staron S: Mammalian skeletal muscle fiber type transition. Int'l Rev Cytol 170: 143-223, 1997

23. Baumann $H$, Jaggi $M$, Solanf $F$, Howald $H$, and Sdhaub $\mathrm{MC}$ : Exercise training induces transitions of myosin isoform subunits within histochemically typed human muscle fibers. Pflugers Archiv 409: 349-360, 1987

24. Demirel HA, Powers SK, Naito H, Hoghes $M$, and Coombes JS: Exercise-induced alterations in skeletal muscle myosin heavy chain phenotype: dose-response relationship. J Appl Physiol 86: 1002-1008, 1999

25. Sullivan V, Powers S, Criswell D, Tumer N, LaRochelle $J$, and Lowenthal D: Myosin heavy chain composition in young rats and old rats skeletal muscle: effects of endurance exercise. J Appl Physiol 78: 2115-2120, 1995

26. Roy RR, Monke SR, Allen DL, and Edgerton VR: Modulation of myonuclear number in functionally overloaded and exercised rat plantaris fibers. J Appl Physiol 87: 634-642, 1999

27. Tseng BS, Kasper CE, and Edgerton VR: Cytoplasm to myonucleus ratios and succinate dehydrogenase activities in adult rat slow and fast muscle fibers. Cell Tissue Res 275: 39-49, 1994

28. Darr KC, and Schultz E: Hindlimb suspension suppresses muscle growth and satellite cell proliferation. J Appl Physiol 67: 1827-1834, 1989 
